\title{
Construction and Evaluation of Normalized cDNA Libraries Enriched with Full-Length Sequences for Rapid Discovery of New Genes from Sisal (Agave sisalana Perr.) Different Developmental Stages
}

\author{
Wen-Zhao Zhou *, Yan-Mei Zhang, Jun-Ying Lu and Jun-Feng Li \\ South Subtropical Crops Research Institute, Chinese Academy of Tropical Agricultural Science, \\ Zhanjiang 524091, China; E-Mails: yanmeizhang2002@163.com (Y.-M.Z.); \\ junyinglusisal@163.com (J.-Y.L.); junfeng1467@163.com (J.-F.L.) \\ * Author to whom correspondence should be addressed; E-Mail: wenzhaozou@163.com; \\ Tel.: +86-0759-2859216; Fax: +86-0759-2859124.
}

Received: 4 July 2012; in revised form: 25 September 2012 / Accepted: 26 September 2012 / Published: 12 October 2012

\begin{abstract}
To provide a resource of sisal-specific expressed sequence data and facilitate this powerful approach in new gene research, the preparation of normalized cDNA libraries enriched with full-length sequences is necessary. Four libraries were produced with RNA pooled from Agave sisalana multiple tissues to increase efficiency of normalization and maximize the number of independent genes by SMART ${ }^{\mathrm{TM}}$ method and the duplex-specific nuclease (DSN). This procedure kept the proportion of full-length cDNAs in the subtracted/normalized libraries and dramatically enhanced the discovery of new genes. Sequencing of 3875 cDNA clones of libraries revealed 3320 unigenes with an average insert length about $1.2 \mathrm{~kb}$, indicating that the non-redundancy of libraries was about $85.7 \%$. These unigene functions were predicted by comparing their sequences to functional domain databases and extensively annotated with Gene Ontology (GO) terms. Comparative analysis of sisal unigenes and other plant genomes revealed that four putative MADS-box genes and knotted-like homeobox (knox) gene were obtained from a total of 1162 full-length transcripts. Furthermore, real-time PCR showed that the characteristics of their transcripts mainly depended on the tight expression regulation of a number of genes during the leaf and flower development. Analysis of individual library sequence data indicated that the pooled-tissue approach was highly effective in discovering new genes and preparing libraries for efficient deep sequencing.
\end{abstract}


Keywords: sisal; normalization; full-length; cDNA library; knox gene; MADS-box gene; expression

\section{Introduction}

As an important economic crop, Agave plants had been exploited and broadly utilized in different fields [1]. The best known modern agave products are tequila and agave sugars as dietary supplements and substitutes for sugar and fats [2,3] or for the production of paper [4]. Recently, some Agave species have been regarded as bioenergy crops [5] because their exploitation for bioenergy production will not divert resources from staple food crop production, as is the case of maize when used for bioenergy production [1]. Because Agave plants have many unique and interesting biological characteristics, they have also attracted the interest of plant physiologists, notably in the field of crassulacean acid metabolism (CAM) and their adaptation to arid climates, in addition to extensive studies by taxonomists [6,7]. Among the Agave species, Agave sisalana is one of the most important species in widespread cultivation and application [8].

Despite the current and potential economic importance and research interests, little basic research had been carried out on these species due to a lack of basic genetic knowledge, large genome size estimated at between 2940 and $4704 \mathrm{Mbp}$ of DNA [9], and long life cycle (5-8 years), especially at the genetic and molecular levels. With comparable species such as pineapple [10], only ca. 310 sequences had been deposited for the whole Agave genus, including 82 sequences from Agave tequilana, which were mainly ribosomal genes, transposon-like sequences and chaperones. Although Simpson et al. had constructed $A$. tequilana cDNA libraries and got some sequence information [1], no large-scale genomic or transcriptomic sequencing data is available in Genbank.

Currently, genetic or breeding studies of intra- and interspecific crosses have been successful [11], but the conventional hybridization was at low efficiencies and high costs in terms of labor, resources and money. In vitro regeneration of most Agave species tested was relatively easy and could be achieved either by indirect organogenesis or through in vitro suspension culture $[8,12]$. However, the asexual propagation led to vulnerability in adverse environmental conditions or attack by pests and pathogens. Based on these reasons, exploring some functional genes will be critical for improving the physiological characterizes and breeding excellent varieties.

Construction of full-length cDNA library and sequencing of ESTs could help in rapid gene discovery, especially in non-model organisms where no prior sequencing data is available. Unfortunately, conventional cDNA libraries not only need high amounts of starting mRNA $(5-100 \mu \mathrm{g})$, but also contain a high percentage of $5^{\prime}$ truncated clones due to the premature stop of reverse transcription (RT), especially large mRNAs tending to form secondary structures [13]. For these reasons, SMART TM technology for full-length enriched cDNA is very straightforward and robust and requires only $0.025-1 \mu \mathrm{g}$ of starting mRNA [14]. This technology utilizes the property of some MMLV reverse transcriptases to add a few $\mathrm{C}$ residues at $3^{\prime}$ end of the first strand cDNA, but not at prematurely terminated reverse transcripts [15]. Moreover, the percentage of full-length clones with the SMART technique is much higher compared with other full-length enriching techniques $[13,16]$. 
Due to differences in the level of gene expression among various cell types, the construction of a normalized cDNA library is necessary in approximately equal quantities and substantially increasing the efficiency of the search for rare genes. However, most of the available approaches based on the re-association of amplified plasmid libraries are not appropriate for long cDNA normalization [17]. Interestingly, duplex-specific nuclease (DSN) from the hepatopancreas of the Kamchatka crab displays a strong preference for cleaving dsDNA and DNA in DNA-RNA hybrid duplexes compared with ssDNA and RNA, irrespective of sequence length. The use of this enzyme allows us to develop a new, simple and effective method for normalization of cDNA, enriched with full-length sequences [18].

To provide insight into transcription characteristics and rare gene functions involved in different development periods of $A$. sisalana, the objective of this study was to construct and characterize the normalized full-length cDNA library combining the robust SMART ${ }^{\mathrm{TM}}$ technique and DSN method. Sequences were evaluated and annotated to classify different categories. By contrast, MADS-box and Knotted1-like homeobox (KNOX) gene families were contained in our libraries. The MADS-box and knox genes had been demonstrated to regulate various aspects of development in all green plant lineages and play a key role in maintaining a pluripotent cell population, called the shoot apical meristem (SAM) [19-25]. Although only a few studies regarding these potential functional genes have been reported for Agave plants, there were no any references in A. sisalana. Here, we report on the isolation and functional analysis of one putative Asknox and four MADS-box genes positioned into three subclasses from sisal. These studies revealed that they could participate in regulating leaf formation and floral development, and helped us to understand how these proteins influence plant development.

\section{Results and Discussion}

\subsection{Generation of the Full-Length Enriched and Normalized cDNA}

By contrast, published protocols did not lead to equal representation among clones of different sizes, maintain the length of long cDNAs after hybridization, or incorporate simultaneous normalization and subtraction of cDNAs $[14,15,26]$. To avoid those problems related to amplification of libraries, exploring a technique to normalize and subtract cDNA before cloning was necessary. In our strategy, the first strand cDNA synthesis was performed in the presence of the SMART ${ }^{\text {TM }}$ RNA oligo in the reaction. And then, the full-length selective step was carried out following cDNA synthesis. Because smaller cDNAs are more preferentially amplified than large cDNAs during PCR [13], more PCR cycles must be done on the large fractions to obtain an equivalent amount of PCR product for cloning. During our experiment operation, 14 cycles were adopted to avoid increasing redundancy and reduce errors introduced by PCR polymerase according to size fraction. By these means, large cDNAs could be amplified as efficiently as smaller ones. As a result, both the first-strand cDNA and the amplified cDNA turned out to be flanked by inverted terminal repeats, which can be applied later for both non-directional and directional cloning of cDNA libraries.

Based on the kinetics of cDNA re-association, DSN normalization differed from the other methods by a separation procedure of the normalized ss-fraction [18], and involved the denaturation and re-association of cDNA with the formation of a normalized ss-fraction and a non-target ds-fraction. 
In addition, DSN was a thermostable enzyme active at $70{ }^{\circ} \mathrm{C}$, so the degradation of ds-fraction was carried out at the same temperature as the cDNA re-naturation. This helped avoid a nonspecific hybridization of cDNA during the DSN treatment, and the loss of transcripts prone to the formation of secondary structures [17]. This method has been applied to analyze mouse transcriptomes and demonstrated successful performance [27]. Normalization led to a 250-fold decrease in the representation of the high-abundant genes and brought the ca. $65 \%-70 \%$ frequency of full-length sequences [15].

\subsection{Characterization of A. sisalana $c D N A$ Libraries}

To rapidly discover some novel genes related to plant development, four normalized and full-length enriched cDNA libraries were constructed from different developing periods of $A$. sisalana tissues. The lengths and fullness ratios of cDNA inserts were investigated by PCR to assess the quality of those full-length enriched cDNA libraries [28]. As expected, most of the cDNA insert sizes ranged from 1 to $2 \mathrm{~kb}$ with an average length of $1.2 \mathrm{~kb}$ from normalized libraries (Figure 1a), which reflected the size distribution of the first-strand cDNAs (Figure 1b). However, no cDNAs longer than $3 \mathrm{~kb}$ were found in these samples. BlastX analysis of the sequences revealed that $35 \%$ (1162 out of 3320 unigenes) of the clones could potentially encode for full-length genes with an average length of $1.8 \mathrm{~kb}$ (Table 1). Redundancy rates were calculated in a clustering analysis of all ESTs generated from the normalized library using the program Megalign (Lasergene, DNAstar, Inc., Madison, WI, USA). In fact, the rate of recovery of unigenes in this study was about $85.6 \%$, which is much higher than the $30 \%$ to $40 \%$ reported from non-normalized cDNA libraries [29,30]. This normalization will greatly help to enrich the library for rare genes, and increase the rate recovery of unigenes and reduce the cost of sequencing by avoiding redundant clones.

Figure 1. Features of normalized cDNA libraries enriched with full-length sequences. (a) Insert size distribution in normalized cDNA library; (b) Agarose gel electrophoresis of non-normalized and normalized amplified SMARTTM-prepared cDNA. Lane 1-2, normalized first-strand cDNA; lane 3-4, non-normalized cDNA; lane M, DL2000 ladder (Takara, Japan).
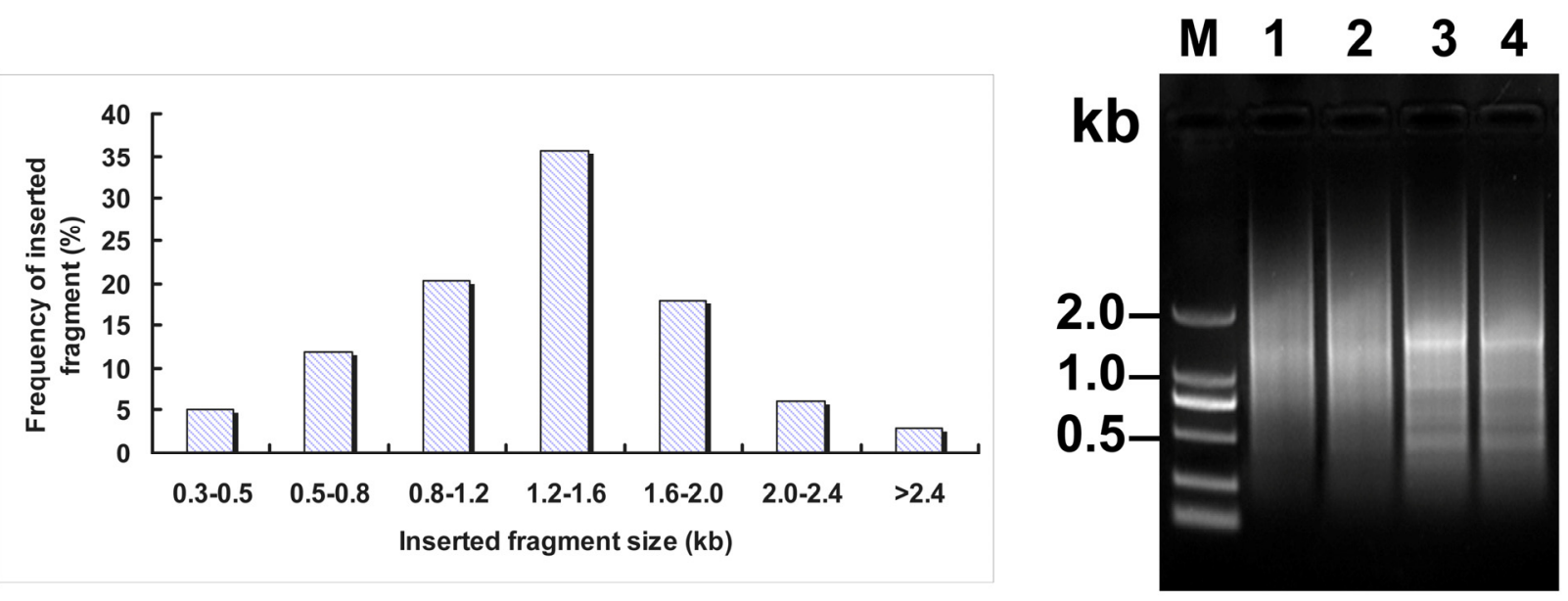

(a)

(b) 
Table 1. Summary of cDNA sisal libraries.

\begin{tabular}{ll}
\hline Description & Number \\
\hline Total number of successful sequences & 3875 \\
Unique sequences & 3320 \\
$5^{\prime}$ end unigenes & 2080 \\
3' end unigenes & 2402 \\
Full-length genes & 1162 \\
Number of known genes & 2158 \\
Unique unknown genes & 1162 \\
\hline
\end{tabular}

From four normalized and full-length enriched cDNA libraries, 4500 clones were randomly selected from the selection medium and sequenced using M13 reverse primer. Successful sequences were 3875. Those clones containing vector backbone and additional sequences that were added during cDNA synthesis were removed. The sequencing results were compared to genes in the non-redundant (nr) protein database using a BlastX search to determine the fullness ratios of the library (Table 1). Of these 3320 sequences, 2158 (65\%) matched known genes, and 67\% of the clones of classified known genes were predicted to contain a putative ATG translation initiation codon $[13,16]$. A total of 3320 putative novel transcripts included a large proportion of singletons $(80.64 \%)$ and a small percentage of contigs $(13.36 \%)$, which had no significant hits to the non-redundant (nr) protein databases of the NCBI. As expected, our libraries were abundant in several non-redundant and full-length sequences, which could be used for efficient deep sequencing in order to explore several rare genes.

\subsection{Gene Ontology Annotation and Bioinformatics Analysis}

In our study, contig assembly was done to remove the redundant ESTs and produce a set of unique, high-fidelity virtual transcripts (unigenes). The partial full-length unigenes were compared to these protein sequences published in the databases using the Blastx program (Table 2). Based on comparation and pfam annotations, A. sisalana unigenes were further annotated for each unigene with Gene Ontology (GO) terms. A total of 3320 unigenes were assigned in the biological process category, molecular function category and cellular component category (Figure 2). Most of the terms in which the $E$-value showed significance were enriched in our unique sequences. This classification provided information on the percentage of unigenes involved in the signal transduction, anabolism, catabolism, reproduction etc. Based on their function in different cellular compartments and anatomical structures, the majority of the unigenes were grouped under "other intra-cellular components", "unknown cellular components", and "other cytoplasmic components", which accounted for about $63 \%$ of the unigenes (Figure 2a). 
Table 2. Putative functions assigned to unigenes from sisal normalized libraries.

\begin{tabular}{lllll}
\hline Library ID $^{\text {a }}$ & Functional annotation $^{\text {b }}$ & $\boldsymbol{E}$-value & Accession No. & Organism species \\
\hline SF1324 & Knotted1-like homeobox protein & $4 \times 10^{-112}$ & CAB88029 & Dendrobium nobile \\
SL980 & NADP-dependent malic enzyme & $1 \times 10^{-91}$ & ABR26037 & Oryza sativa \\
SL712 & cytochrome P450 like_TBP & $3 \times 10^{-95}$ & BAA10929 & Nicotiana tabacum \\
SL349 & ATP synthase subunit beta & $6 \times 10^{-122}$ & XP003627732 & Medicago truncatula \\
SF1073 & unknown protein & $2 \times 10^{-133}$ & ACU14517 & Glycine max \\
SF1130 & MADS-box transcription factor & $4 \times 10^{-155}$ & ABC70707 & Asparagus virgatus \\
SL453 & chloroplast photosystem & $1 \times 10^{-37}$ & ACZ54010.1 & Wolffia arrhiza \\
SL324 & Sucrose synthase & $8 \times 10^{-104}$ & XP003591492 & Medicago truncatula \\
SS239 & Disease resistance protein & $2 \times 10^{-45}$ & XP002275269 & Vitis vinifera \\
SS136 & UDP-glucosyltransferase & $2 \times 10^{-80}$ & NP001154307 & Arabidopsis thaliana \\
& Ribulose-1,5-bisphosphate & & & \\
SF201 & carboxylase & $3 \times 10^{-180}$ & AFA55129 & Agave schottii \\
SS712 & Conserved hypothetical protein & $2 \times 10^{-110}$ & BAD94036 & Arabidopsis thaliana \\
SL506 & Methionyl-tRNA synthetase & $4 \times 10^{-189}$ & AAC99620 & Oryza sativa \\
SF321 & Sugar transporter & $2 \times 10^{-98}$ & CAA90628 & Arabidopsis thaliana \\
SF603 & Poly(A)-binding protein & $4 \times 10^{-110}$ & CAC01238 & plumbaginifolia \\
\hline
\end{tabular}

a: The library ID is indicated by the first letter of the designation: $\mathrm{S}$, sisal and the second letter of the designation: S, stem; L, leaf; F, flower. ${ }^{b}$ : Similarity gene function was conducted using the BLASTX program.

Figure 2. Functional categorization of unigenes with Gene Ontology (GO) terms at The Arabidopsis Information Resource (TAIR). These results in the unigenes were functionally classified under three main functional categories: cellular component (a), molecular function (b) and biological process (c) with respective GO Slim terms.

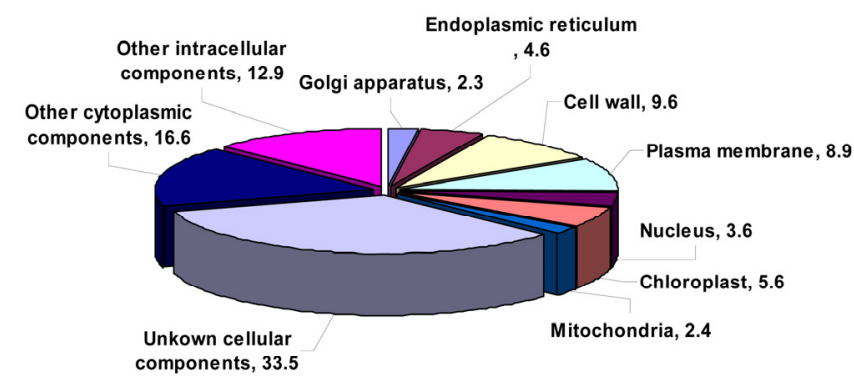

(a)

Go Slim terms - GO Cellar component

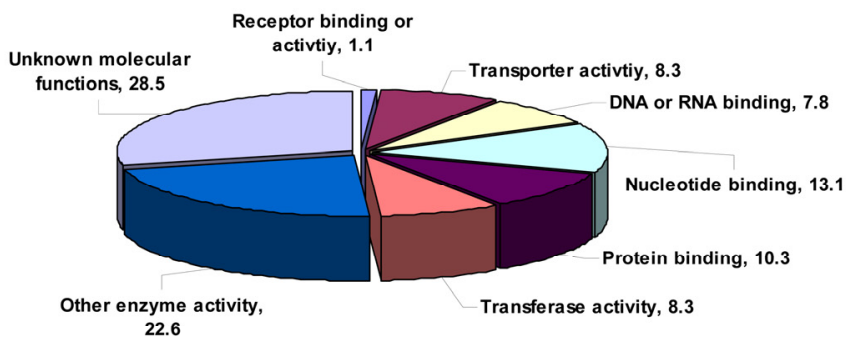

(c)

Go Slim terms - GO Molecular function

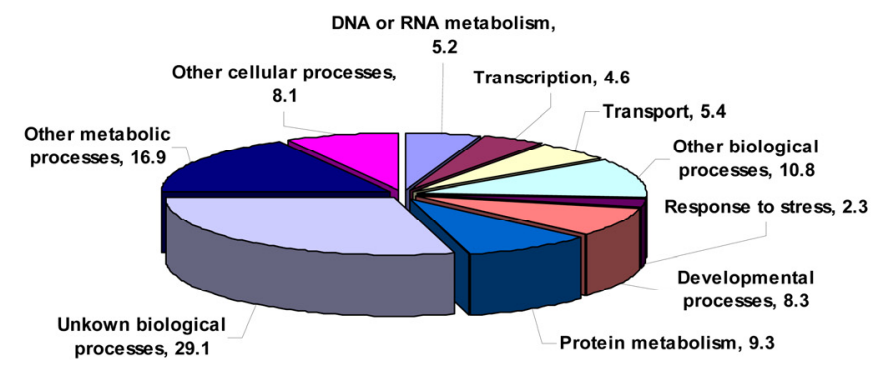

(b)

Go Slim terms - GO Biological process 
In contrast to their biological functions, the unigenes were then classified into nine different metabolism processes and an unknown item. The larger part of the unigenes were divided into "other cellular process", "other biological process", "other metabolic process" and "unknown biological processes" accounting for $8.1 \%, 10.8 \%, 16.9 \%$ and 29.1\%, respectively (Figure 2b). In addition, a large number of $A$. sisalana unigenes appeared to be involved in plant molecular functions, such as transporter activity, kinase activity, transferase activity, or nucleotide and protein binding activities (Figure 2c). Of the unique sequences, it was indicated that the normalization and identification of new functional genes from our full-length cDNAs were very efficient.

\subsection{Tissue-Specific Gene Expression}

\subsubsection{Asknox Expression Characteristics Related to Organogenesis}

Plant leaves develop as flat lateral organs from SAM, and the establishment of polarity along three-dimensional axes (the proximodistal, mediolateral and adaxial-abaxial axes) is crucial for the growth of normal leaves [20], especially for sisal. In our study, a knotted-like homeobox (knox) gene (Asknox, $1074 \mathrm{bp}$ ) encoded homeodomain-containing transcription factor was obtained from the early developmental cDNA library of A. sisalana. Homology comparison, AsKNOX protein sequence shares $57 \%$ with $A$. thaliana KNAT2 and KNAT6 (ID: NP850951), 53\% identity with Solanum lycopersicum TKN (ID: Q9ZRC0), and 51\% with closely-related monocot genes, O. sativa OSH6 (ID: BAA79224). As with other species, AsKNOX possesses all three highly conserved domains typical to the KNOX proteins: the MEINOX domain that is subdivided into KNOX1 and KNOX2 (Figure 3a), the ELK domain and the homeodomain (Figure 3b). AsKNOX also contained a GSE domain between the MEINOX and ELK domains, similar to previous reports [31]. It is therefore reasonable to assume that AsKNOX protein possesses DNA-binding activity via its homeodomain, dimerization activity via its MEINOX domain, a nuclear localization signal via its ELK domain, and a protein degradation signal via its GSE domain [31,32].

Figure 3. Sequence alignment and phylogenetic tree of the AsKNOX protein selected relatives from other plant species. (a) Schematic diagram of the AsKNOX protein structure by Position-Specific Iterated Blast; (b) Alignment of the deduced amino acid sequences of AsKNOX and KNOXs from A. thaliana (P46639), Pisum sativum (O82805) and Prunus persica (ABO28750). Identical and conserved amino acid residues were labeled in various colors, respectively. The different functional domains were indicated by black lines above the corresponding sequences. Dashes indicated gaps introduced to optimize the alignment. Sequences were aligned using the CLUSTALX program of DNAman; (c) Relationship tree of AsKNOX and its relatives in the class I KNOX group. The tree was obtained using the neighbor-joining method performed with the amino acid sequence alignment of the region comprising the MEINOX, GSE and HD domains. To assess support for the inferred relationships, 1000 bootstrap samples were generated. Sequences were designated by accession numbers and the name of organisms from the GenBank database. One Class II KNOX protein from M. truncatula was used as outgroup. 


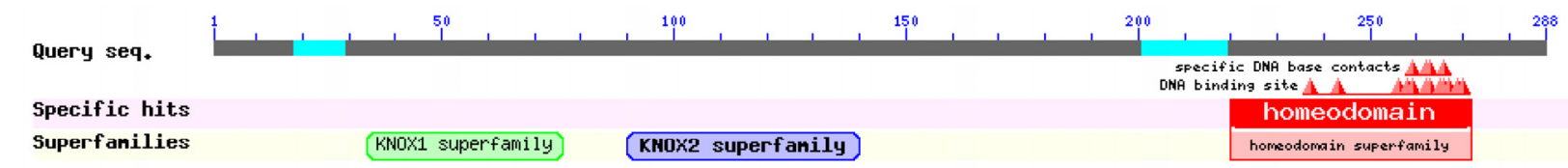

(a)

MEINOX

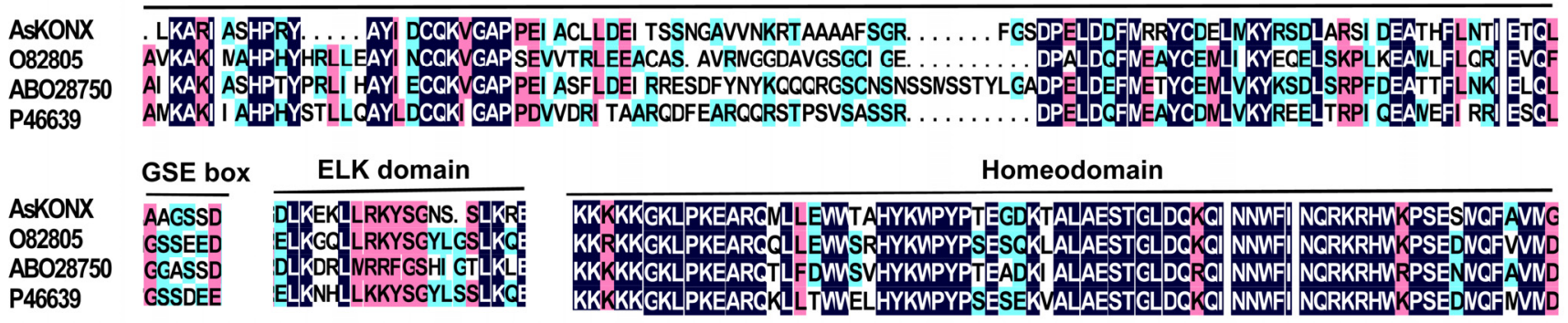

(b)

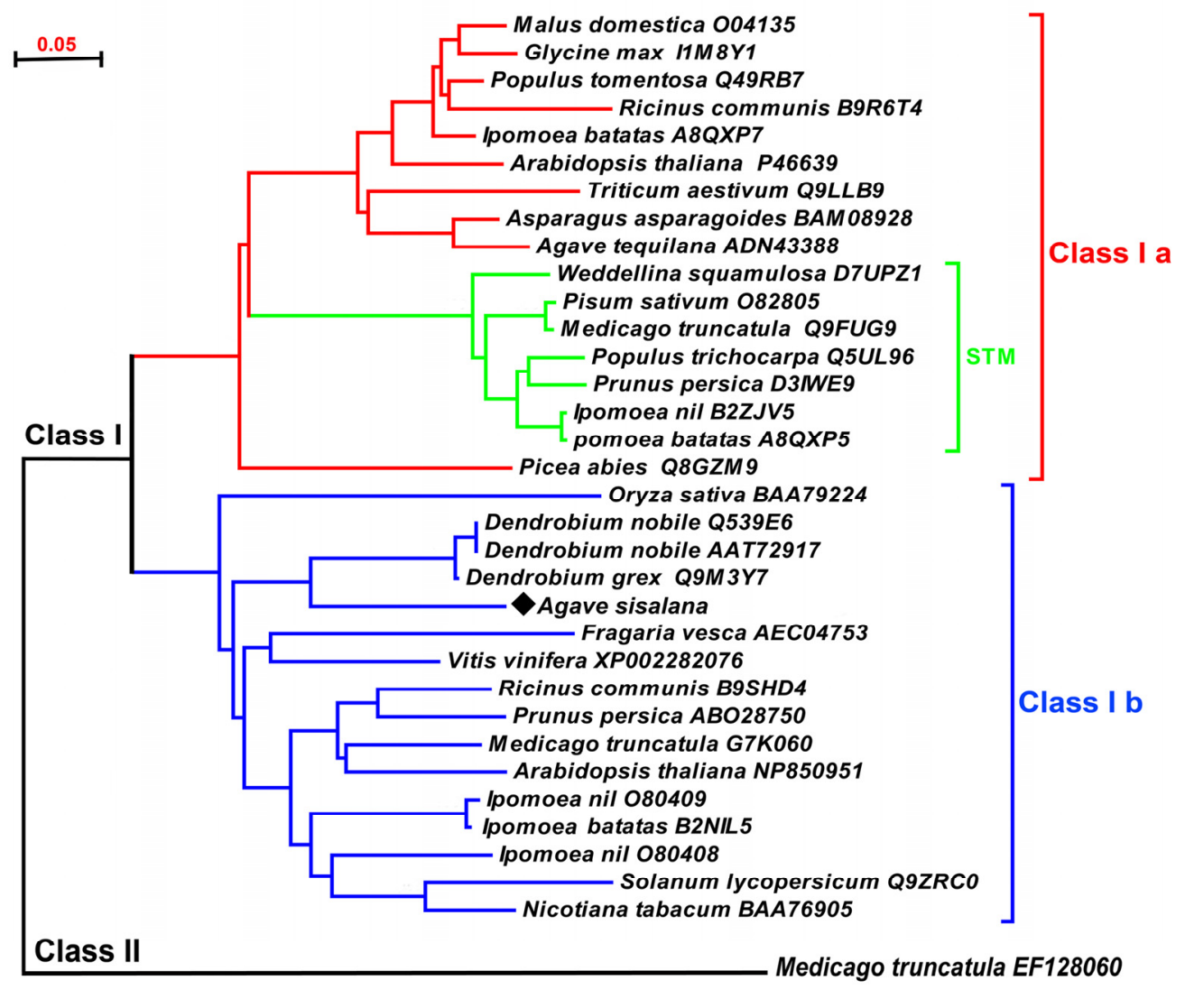

A phylogenetic comparison of class I KNOX proteins, including that of $A$. sisalana, using amino acid sequences covering the MEINOX-HD region, enables the subdivision of the protein family into two main clades, namely Classes Ia and Ib (Figure 3c). The global tree topology reveals that KNOX protein sequences from diverse species positioned AsKNOX with class Ib KNOX proteins. Though monocots and dicots are all found in two subclasses, distinct boundaries can be distinguished between them (Figure 3c). On the basis of these relationships, it can be postulated that at least one duplication in the ancestral class I gene occurred before the monocot-eudicot split, leading to the divergence of class Ia and class Ib groups of genes [33]. 
Figure 4. Real-time PCR analysis of Asknox gene expression profiles in different leaf and inflorescence development stages. (a) Analysis of the Asknox expression profiles in apical meristem and leaves during different development stages; (b) Analysis of the Asknox expression profiles in different development stages of inflorescence; (c) Analysis of the Asknox expression profiles in different organs of inflorescence; (d) Schematic diagram of different development stages and organs of inflorescence.

(a)
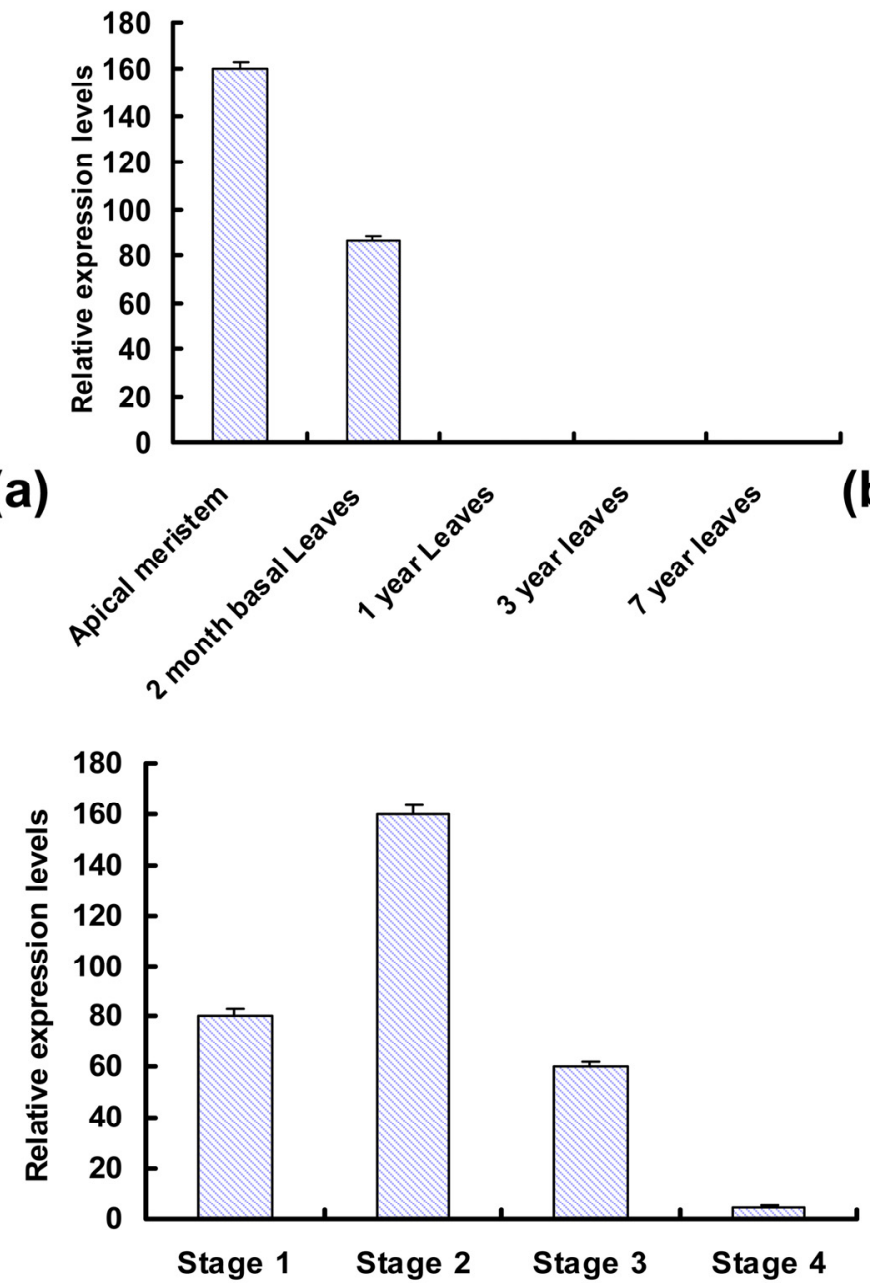

(c)

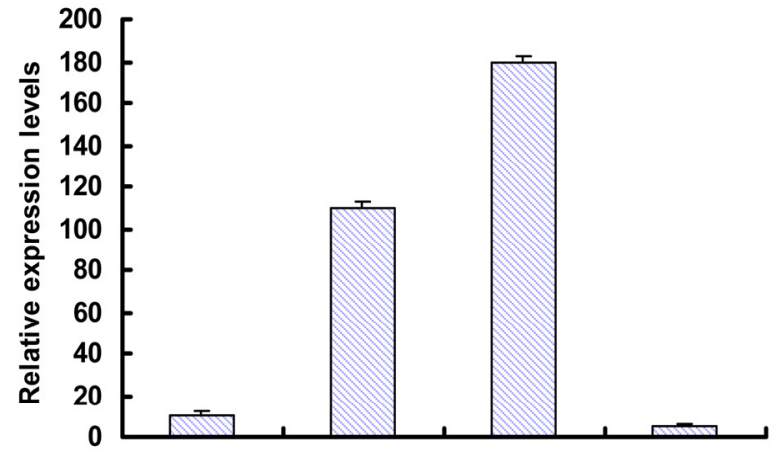

(b)
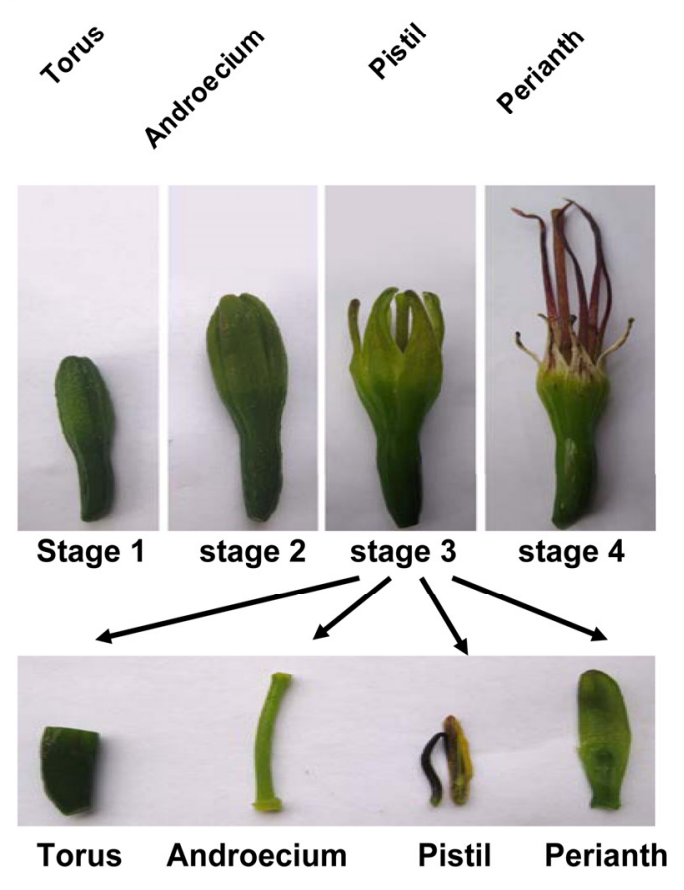

To understand transcription characteristics of Asknox, real-time PCR was performed in different development stages of leaf and flower. Asknox transcripts were mainly expressed in the vegetative shoot apex containing the single SAM and also in the developing inflorescence (Figure 4). Interestingly, Asknox was strongly expressed in the apical meristem and at relative low levels in leaf tissues, and could not be detected in grown-up leaves with analogous expression reports of KNOX family genes in other species [20,22]. However, the transcription expression is still detectable in early developing leaves (Figure 4a). It was postulated that Class I Asknox expression might correlate with sisal leaf shape, like simple-leafed species, such as $A$. thaliana and maize [34,35]. Ten years after knox overexpression was first shown to increase tomato leaf complexity, genetic evidence was provided that KNOX activity was necessary and sufficient for leaflet formation in C. hirsuta [36]. A similar explanation had been offered for the variable leaf phenotypes observed in transgenic tobacco plants 
constitutively expressing the tobacco konx genes, TKN1 and TKN2 [37]. Loss-of-function and over-expression of knox genes had serious effects on shapes and sizes of leaves. The reason is that KNOX proteins may regulate hormone levels and repress the transcription of the IAA and GA-synthetic genes, and have impressive effects on leaf morphology [38,39]. These results suggested that knox transcription factors drive leaf development in various ways in different species by controlling the temporal action of cellular growth and differentiation pathways during early stages of the leaf.

In addition, Asknox transcripts were also readily detectable in early developing floral buds with high levels in pistil and androecium, but barely detectable in torus and perianth (Figure 4b). Asknox expression was increased 2-fold in developing floral buds with early initiating petal-spur primordial (Figure 4c), and supports the hypothesis that Asknox may also have a role in keeping normal patterning in these tissues. This hypothesis was identified by analysis of transgenic plants in Arabidopsis and strawberry, where defects in flower form, petal abscission, fruit set, and fertility were observed [25]. Thus along with leaf development, Asknox plays a significant role in floral architecture and function, consistent with it relatively high level of expression in these tissues.

\subsubsection{Expression Characteristics of $M A D S-B o x$ Gene Family Related to Floral Development}

A search for gene sequences in the A. sisalana transcriptome database, four potentially distinct $M A D S$ genes (AsMADS-box 1, 2, 5 and 6 ) including the putative complete open reading frames were determined. The alignment analysis showed that AsMADS-box1, 2, 5 and 6 shared with amino acid identities of $90 \%, 84 \%, 82 \%$ and $91 \%$ to MADS-boxl (AEX92976), MADS-box2 (AEX92975), MADS-box5 (AEX92969) and MADS-box6 (AEX92972) from A. tequilana, respectively (Supplementary data, Figure S1). As most plant MADS-box sequences, four homology proteins from A. sisalana fell into the stereotypical type II category on the basis of the full-length amino acid sequences. Further analysis showed that four genes belonged to three subfamilies, such as AGL2, STMADS11 and DEF, respectively (Table 3, Figure 5a). Highly conserved amino-acid consensus sequences were also found in their MADS-box domains (Figure 5b). A coiled-coil structure ( $K$ domain) also appeared. In addition, the $M A D S$-box and $K$ domains were separated by a weakly conserved intervening domain ( $I$ domain) (data not shown). It had been shown that the $I$ and $K$ domains were involved in protein-protein interactions [40].

Table 3. Functional prediction of $M A D S$-box genes from A. sisalana compared with Arabidopsis.

\begin{tabular}{cccccc}
\hline Gene name & Orthologous gene $^{\text {a }}$ & E-value & $\begin{array}{c}\text { Max } \\
\text { identity }\end{array}$ & $\begin{array}{c}\text { Gene } \\
\text { subfamily }\end{array}$ & Functional role predicted \\
\hline MADS-box1 & $\begin{array}{c}\text { NP_564214 } \\
\text { (SEPALLATA 3) }\end{array}$ & $5 \times 10^{-108}$ & $67 \%$ & AGL2 & E function \\
MADS-box2 & $\begin{array}{c}\text { NP_186880 } \\
\text { (SEPALLATA 2) }\end{array}$ & $1 \times 10^{-82}$ & $60 \%$ & AGL2 & E function \\
MADS-box5 & NP_179840(SVP) & $2 \times 10^{-90}$ & $61 \%$ & STMADS11 & $\begin{array}{c}\text { Control of flowering time } \\
\text { Class B floral homeotic } \\
\text { MADS-box6 }\end{array}$ \\
$\begin{array}{c}\text { NP_191002 } \\
\text { (APETALA 3) }\end{array}$ & $7 \times 10^{-69}$ & $51 \%$ & DEF & \begin{tabular}{c} 
gene \\
\hline
\end{tabular}
\end{tabular}

${ }^{\mathrm{a}}$ : The classification was based on the Arabidopsis transcription database [40]. 
Figure 5. Phylogenetic tree and $C$-terminal domain alignment of the deduced MADS-box protein sequences from $A$. sisalana. The neighbor-joining tree had been constructed using a representative subset of 58 sequences from available plant MADS-box sequences (a). These 58 sequences have been selected as follows: To assess support for the inferred relationships, 1000 bootstrap replications were generated. Alignment of $C$-terminal amino acid domains of A. sisalana MADS proteins (b) based on motifs previously identified by Vandenbussche et al. [41]. Sequences are designated by accession numbers and organism names from GenBank database.

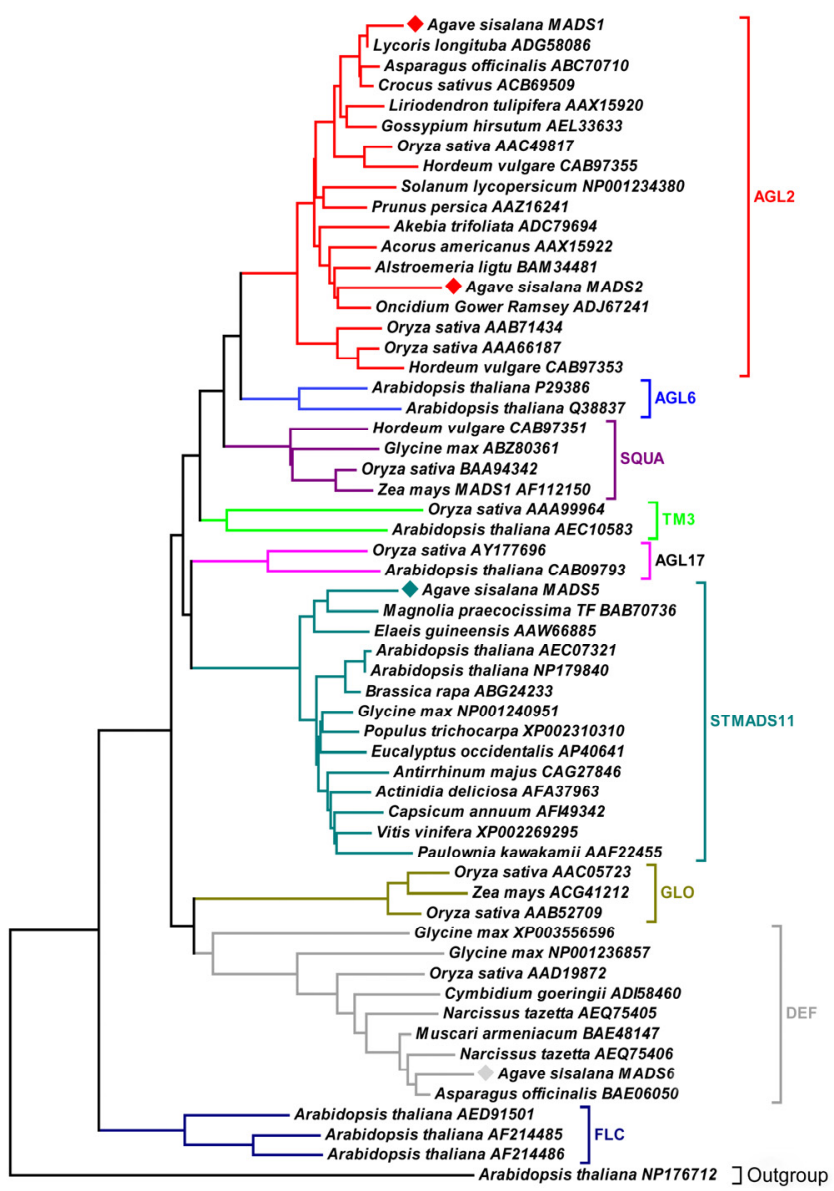

(a)

$※$ MADS1
ABC70710
ADG58086
NM001125758
AAC49817
AAX15920
AEL33633

※MADS2
AAX15922
AAB71434
ADC79694
BAM34481
ADJ67241
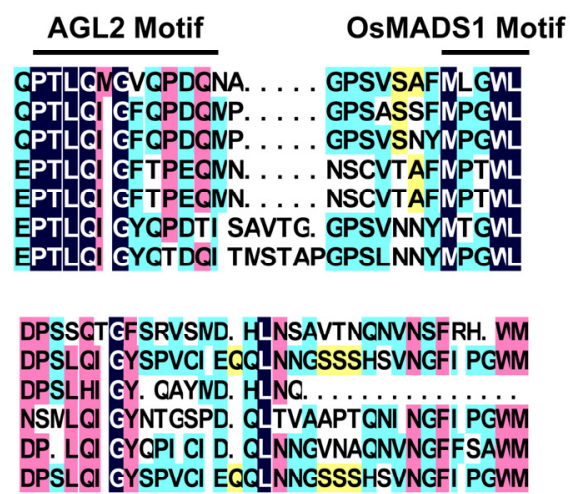

STMADS11 Motif

※MADS5 XP002269295 AFA37963 AEC07320 AAW66885 NP001240951 BAB70736

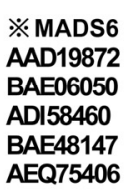

(b)

Although the MADS-box genes had been extensively investigated and shown to be essential for inflorescence and flower development in many model plants [23,24], there was an infrequent research in A. sisalana. On the basis of protein sequence alignment, AsMADS-boxl and 2 were closely related to SEPALLATA3 (SEP3) and SEPALLATA2 (SEP2) within the E function genes, which were required for the formation of petal, stamen and carpel [19]. Interestingly, in our study, real-time PCR revealed that the parallel expression patterns showed between the AsMADS-box 1 and 2, although AsMADS-box 1 had more highly expressed levels compared with AsMADS-box2 (Figure 6a, b). In inflorescence tissues, the high levels of expression were observed for MADS-box 1 and 2 at the initial stages of bud development and decreased during flower formation; however, no expression was detected in fully developed leaf tissue. These results were similar to SEP-like and OSMADS1-like gene expression patterns in orchid and $A$. tequilana, which were detected in inflorescences and developing 
flowers $[42,43]$. This was predicted that they might encode proteins of redundant functions based on homology analysis with Arabidopsis [44]. The significant fold changes of expression levels between $M A D S$-box 1 and 2 of E function genes suggested that MADS-box 1 need to be further analyzed in order to demonstrate the role in the floral transition and development in A. sisalana, and may also reflect the diversity in the E group genes in various plant species during evolution.

Figure 6. Real-time PCR analysis of AsMADS-box gene expression profiles in different tissues and inflorescence development stages. (a) AsMADS-box 1 gene expression profiles; (b) AsMADS-box 2 gene expression profiles; (c) AsMADS-box5 gene expression profiles; (d) AsMADS-box6 gene expression profiles.
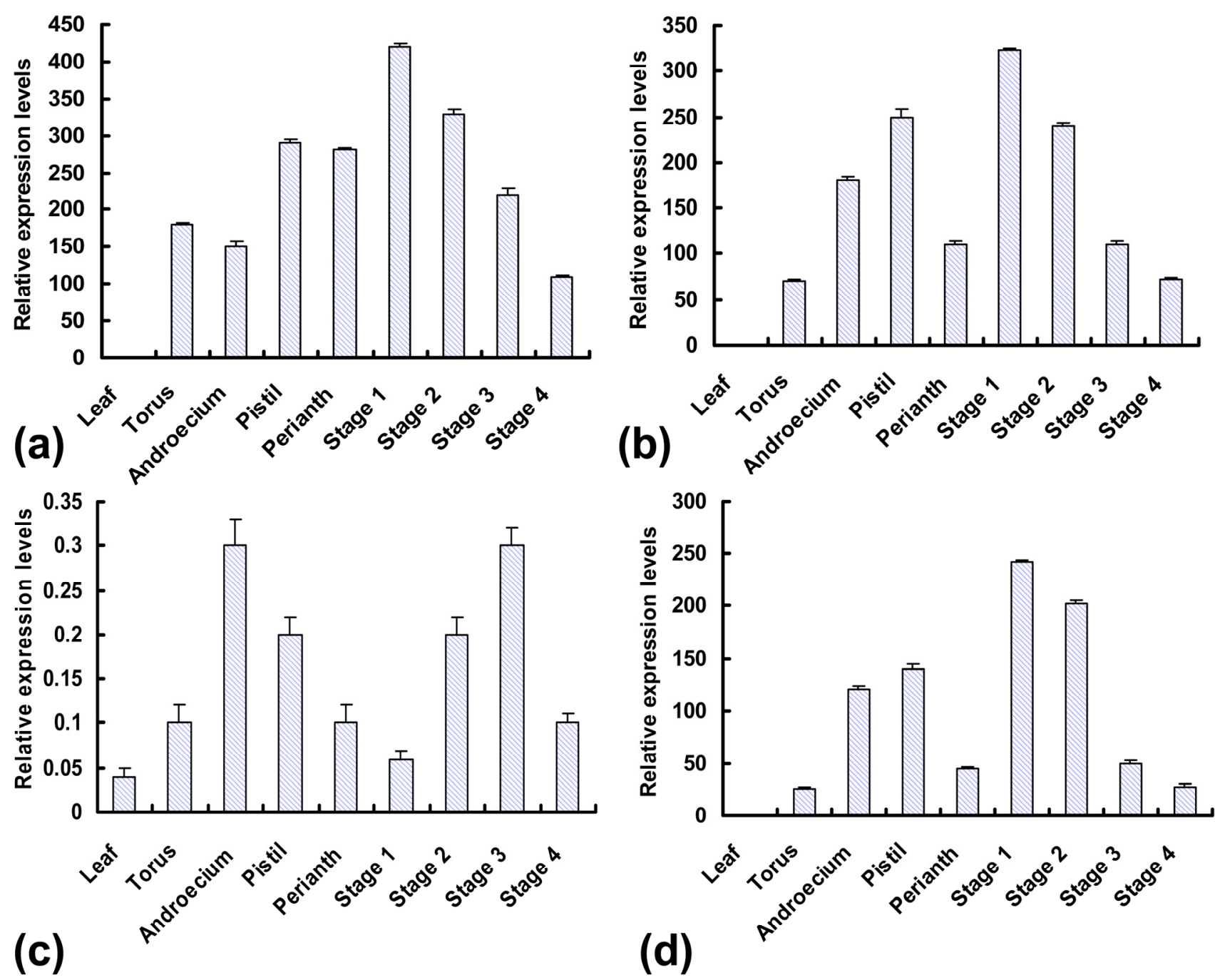

In addition, interactions between the proteins encoded by the ABC type genes and the SEP-like genes were essential for the correct regulation of flower development. This assumption was further supported by the interaction between SEP and B, C function proteins [45]. For example, AP3 and PI had been shown to regulate petal and stamen development by interacting with SEP1, SEP2, and SEP3 $[19,45,46]$. This result strongly indicated that E function genes were necessary for the activities of the $\mathrm{B}$ and $\mathrm{C}$ function genes. 
However, MADS-box5 showed no significant differences in expression levels between any flower organs and developmental stages (Figure 6c), because StMADS11-like genes had been shown to play an important role in the vegetative to floral transition as in the case of SVP from $A$. thaliana that repressed the switch from vegetative to floral growth [47,48]. The svp mutants of $A$. thaliana flowered earlier due to a reduced vegetative growth phase, and also passed more rapidly during the different stages of vegetative growth [49].

Based on the pattern of MADS-box6 ( $D E F$-like) expression with similar expression profiles to AsMADS-box2 gene, it was conceivable that this gene could also exert some effect on flower formation (Figure 6d). In A. thaliana, heterodimers form between DEF and GLO proteins, which in turn interact with SEP-like proteins to form a functional unit. Similar homodimerization was also found in tulip [50], although the functionality of these complexes in specific floral organs was unclear. Sandoval et al. reported that the A. tequilana DEF-like and GLO-like genes show disproportionate changes in expression levels due to homodimerization formation of DEF-like protein [43]. In A. sisalana, whether the heterodimerization and homodimerization are produced between AsMADS and GLO-like proteins and interact with SEP-like proteins still need to be further investigated.

The data presented here show that MADS factors play different roles in the developmental pathway that finally leads to plant reproduction. Due to the complex genetic background in A. sisalana, how the MADS-box genes regulate the diverse developmental processes ranging from root to flower and fruit development is still further explored using the mutated and transgenic plants. With the gene functions elaborated in some exciting models and the novel genes discovered using the normalized cDNA library, those will considerably contribute to a better understanding of the relationship between functional genes and plant development.

\section{Experimental Section}

\subsection{Plant Materials}

Field-grown sisals (Agave sisalana Perr.) were obtained from the Sisal Field Germplasm Bank of the Southern Subtropical Crop Research Institute of CATAS (Guangdong, China). Four development stages of different tissues (SAM, root, stem, flower, and leaf) were randomly sampled from two month seedlings in the greenhouse to one, three, and seven year plants in the field. These sliced tissues of three independent plants were pooled together as one of three replications at each sampling stage. All of the tissues were immediately frozen in liquid nitrogen and stored at $-80{ }^{\circ} \mathrm{C}$ before being analyzed.

\subsection{Poly $\left(A^{+}\right)$RNA Isolation and First-Strand cDNA Synthesis}

Total RNAs isolated from different tissues using Trizol reagent (Life Technologies Inc., Invitrogen, Carlsbad, CA, USA) were assessed by absorbance at $260 \mathrm{~nm}$ and $280 \mathrm{~nm}$ and agarose gel electrophoresis (1.5\%), respectively. For construction of those full-length enriched cDNA libraries,

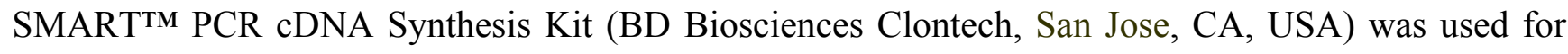
the synthesis of cDNA starting from 0.5 to $1 \mu \mathrm{g}$ of poly $\left(\mathrm{A}^{+}\right) \mathrm{RNA}$ according to the manufacturer's instruction. The primers were the BD Biosciences oligonucleotides SMART TM Oligo VI and CDS-3M containing the $S f i \mathrm{I}$ A and $S f i$ B recognition sequence, respectively (Supplementary data, Table S1). 


\subsection{Amplification of cDNA by Long-Distance Polymerase Chain Reaction}

To prepare the full-length normalized cDNA, the first strand cDNA was amplified with PCR primer provided in the SMART ${ }^{\mathrm{TM}}$ PCR cDNA Synthesis Kit (Clontech). The PCR mixture $(50 \mu \mathrm{L})$ contained $1 \times$ Advantage 2 Polymerize mix, $1 \times$ Advantage 2 PCR reaction buffer, $200 \mathrm{mM}$ dNTPs, $0.3 \mathrm{mM}$ primer and $3 \mathrm{ng}$ first-strand cDNA. Fourteen PCR cycles $\left(95{ }^{\circ} \mathrm{C}\right.$ for $7 \mathrm{~s}, 65^{\circ} \mathrm{C}$ for $20 \mathrm{~s}$, and $72{ }^{\circ} \mathrm{C}$ for $3 \mathrm{~min}$ ) were performed. The samples were used for the DSN normalization and the preparation of cDNA libraries.

\subsection{First-Strand cDNA Normalization and Amplification}

Upon completion of first-strand cDNA synthesis, the reaction mixture was purified using the Qia Quick PCR Purification Kit (Qiagen, Tokyo, Japan), and dissolved in milliQ water to a final cDNA concentration of $100 \mathrm{ng} / \mu \mathrm{L}$ after precipitation with ethanol. The reaction mixture $(1.5 \mu \mathrm{L}$ aliquot, $200 \mathrm{mM}$ Hepes, $\mathrm{pH} 7.5,2 \mathrm{M} \mathrm{NaCl}$, and $0.8 \mathrm{mM}$ EDTA) was denatured at $98{ }^{\circ} \mathrm{C}$ for $3 \mathrm{~min}$ and allowed to hybridize at $70{ }^{\circ} \mathrm{C}$ for $5 \mathrm{~h}$. After re-naturation, $5 \mu \mathrm{L}$ of $2 \times \mathrm{DSN}$ buffer $(100 \mathrm{mM}$ Tris- $\mathrm{HCl}$ $\mathrm{pH} 8.0,10 \mathrm{mM} \mathrm{MgCl}$, and $2 \mathrm{mM}$ dithiothreitol), preliminarily preheated to $70{ }^{\circ} \mathrm{C}$, was added to cDNA samples for $10 \mathrm{~min}$. Then, $0.25 \mathrm{Kunitz}$ units of DSN enzyme (Evrogen, Russia) were added to the reaction mixture for $20 \mathrm{~min}$ at $70^{\circ} \mathrm{C}$, and were subsequently inactivated by the addition of $10 \mu \mathrm{L}$ of $5 \mathrm{mM}$ EDTA.

To amplify the normalized ssDNA fraction after DSN treatment, PCR was carried out in a $50 \mu \mathrm{L}$ reaction mixture using an advantage 2 PCR kit (BD Biosciences Clontech, United States) containing $2 \mu \mathrm{L}$ of reaction mixture, $1 \times$ Advantage 2 Polymerize mix (Clontech), $1 \times$ Advantage 2 PCR reaction buffer (Clontech), $200 \mathrm{mM}$ dNTPs and $0.3 \mathrm{mM}$ CapM primer, which corresponds to the external part of the flanking cDNA adapter. To obtain amplified cDNA samples with a concentration of ca. $20 \mathrm{ng} / \mu \mathrm{L}$, twenty PCR cycles $\left(95{ }^{\circ} \mathrm{C}\right.$ for $7 \mathrm{~s}, 65^{\circ} \mathrm{C}$ for $20 \mathrm{~s}$, and $72{ }^{\circ} \mathrm{C}$ for $3 \mathrm{~min}$ ) were performed.

\subsection{Construction and Quality Analysis of cDNA Library}

Normalized cDNA samples digested by SfiI were cloned into the pDNR-LIB vector (Promega Corp., Madison, WI, USA) with T4 DNA liganse (MBI Ferments Inc., Vilnius, Lithuania), which were used for Escherichia coli (JM109) electro-transformation with the Gene Pulser II system (Bio-Rad company, Richmond, CA, USA) at $1500 \mathrm{~V}$. After shaking for $1 \mathrm{~h}$ at $120 \mathrm{rpm}$ and $37{ }^{\circ} \mathrm{C}$, $200 \mu \mathrm{L}$ of the electroporated cell were spread on Luria-Bertani (LB) agar plate containing $30 \mu \mathrm{g} / \mathrm{mL}$ of chloramphenicol. Frequencies of the corresponding cDNA sequences in the libraries were calculated from the number of positive colonies. For analysis of the insert size distribution, 200 colonies from each library were randomly picked, and were used for PCR with standard M13 primers (Supplementary data, Table 1). The reaction system was followed by denaturation at $94{ }^{\circ} \mathrm{C}$ for $10 \mathrm{~min}$, 30 cycles $\left(94{ }^{\circ} \mathrm{C}\right.$ for $30 \mathrm{~s}, 52{ }^{\circ} \mathrm{C}$ for $30 \mathrm{~s}$, and $72{ }^{\circ} \mathrm{C}$ for $2 \mathrm{~min}$ ), and $72{ }^{\circ} \mathrm{C} 10 \mathrm{~min}$. PCR products were visualized on a $1.2 \%$ agarose gel, following ethidium bromide (EB, $0.5 \mathrm{pg} / \mathrm{mL}$ ) staining, alongside a $1 \mathrm{~kb}$ DNA ladder (Takara, Japan). 


\subsection{Sequence Processing and Analysis}

Vector-derived and ambiguous sequences were eliminated using online software (vecscreen) from NCBI. The EST sequences were clustered and assembled into contiguous consensus sequences (contigs) using the program Seqman and Megalign of DNAstar software. The non-redundant sequences were searched against protein databases obtained from NCBI with a search threshold of $E<1.0 \times 10^{-5}$. All similarity searches were executed using the BlastN, BlastX, and tBlastX tools. Phylogenetic trees were constructed by the Neighbor-Joining (NJ) method using the NJ algorithm implemented in the Molecular Evolutionary Genetics Analysis (MEGA) software version 5.0. The Blast results were used to obtain further information on the function and motif through the InterPro member databases [51].

\subsection{Gene Expression Analysis by Real-Time PCR}

Quantitative PCR was performed by using the first strand cDNA as templates on a Lightcycler (Roche Diagnostics), with the Light Cycler Fast Start Reaction Mix MasterPLUS SYBR Green according to the manufacturer's recommendations. Cycling conditions were as follow: $95{ }^{\circ} \mathrm{C}$ for $5 \mathrm{~min}, 40$ cycles at $95{ }^{\circ} \mathrm{C}$ for $10 \mathrm{~s}, 54{ }^{\circ} \mathrm{C}$ for $30 \mathrm{~s}$, and $72{ }^{\circ} \mathrm{C}$ for $30 \mathrm{~s}$. Expression of $18 \mathrm{~s}$ rRNA was used as an internal control to normalize the amount of mRNA. The data shown represent means of values obtained from three independent biological replicates.

\section{Conclusions}

Four sisal cDNA libraries enriched with full-length sequences were constructed by uniting

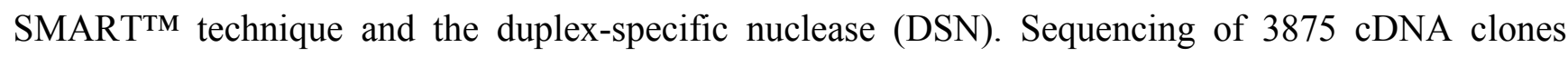
revealed 3320 unigenes (85.7\%) with an average insert length about $1.2 \mathrm{~kb}$, which were extensively annotated with Gene Ontology (GO) terms. This DSN normalization greatly helped to enrich the library for rare genes and increase the rate recovery of unigenes. Furthermore, real-time PCR showed that the transcript characteristics of four putative MADS-box genes and one knotted-like homeobox (knox) gene mainly depended on the tight expression regulation of a number of genes during the flower and leaf development, respectively. Comparative analysis revealed that the pooled-tissue approach was highly effective in discovering new genes and preparing libraries for efficient deep sequencing.

\section{Acknowledgments}

This research was supported by the earmarked fund for China Agriculture Research System (CARS-19).

\section{Conflict of Interest}

The authors declare no conflict of interest. 


\section{References}

1. Simpson, J.; Hernández, A.M.; Juárez, M.J.A.; Sandoval, S.D.; Villarreal, A.S.; Romero, C.C. Genomic resources and transcriptome mining in Agave tequilana. GCB Bioenergy 2011, 3, 25-36.

2. Gomez, E.; Tuohy, K.M.; Gibson, G.R.; Klinder, A.; Costabile, A. In vitro evaluation of the fermentation properties and potential prebiotic activity of Agave fructans. J. Appl. Microbiol. 2009, 108, 2114-2121.

3. Leach, J.D.; Sobolik, K.D. High dietary intake of prebiotic inulin-type fructans in the prehistoric Chihuahuan Desert. Br. J. Nutr. 2010, 103, 1558-1561.

4. Jaouadi, M.; Msahli, S. A study of the physical and mechanical properties of paper made from Agave americana L. fibers. Mater. Manuf. Process. 2011, 26, 567-572.

5. Somerville, C.; Youngs, H.; Taylor, C.; Davis, S.C.; Long, S.P. Feedstocks for lignocellulosic biofuels. Science 2010, 329, 790-792.

6. Wang, D.; Hechathorn, C.A.; Barua, D.; Joshi, P.; Hamilton, E.W.; Lacroix, J.J. Effects of elevated $\mathrm{CO}_{2}$ on the tolerance of photosynthesis to acute heat stress in $\mathrm{C}_{3}, \mathrm{C}_{4}$, and CAM species. Am. J. Bot. 2008, 95, 165-176.

7. Lujan, R.; Lledias, F.; Martinez, L.M.; Barreto, R.; Cassab, G.I.; Nieto-Sotelo, J. Small heat-shock proteins and leaf cooling capacity account for the unusual heat tolerance of the central spike leaves in Agave tequilana var. Weber. Plant Cell Environ. 2009, 32, 1791-1803.

8. Nikam, T.D.; Bansude, G.M.; Aneesh Kumar, K.C. Somatic embryogenesis in sisal (Agave sisalana Perr. ex. Engelm). Plant Cell Rep. 2003, 22, 188-194.

9. Moreno-Salazar, S.F.; Esqueda, M.; Martinez, J.; Palomino, G. Nuclear genome size and karyotype of Agave angustifolia and A. rhodacantha from Sonora, Mexico. Rev. Fitotec. Mex. 2007, 30, 13-23.

10. Antony, E.; Taybi, T.; Courbot, M.; Mugford, S.T.; Smith, J.A.; Borland, A.M. Cloning, localization and expression analysis of vacuolar sugar transporters in the CAM plant Ananas comosus (pineapple). J. Exp. Bot. 2008, 59, 1895-1908.

11. Escobar-Guzmán, R.; Zamudio-hernández, F.; Gil-Vega, K.; Simpson, J. Seed production and gametophyte formation in Agave tequilana and Agave Americana. Bot. 2008, 86, 1343-1353.

12. Flores-Benitez, S.; Jimenez-Bremont, J.F.; Rosales-Mendoza, S.; Arguello-Astorga, G.R.; Castillo-Collazo, R.; Alpuche-Solis, A.G. Genetic transformation of Agave salmiana by Agrobacterium tumefaciens and particle bombardment. Plant Cell Tissue Organ Cult. 2007, 91, 215-224.

13. Wellenreuther, R.; Schupp, I. The German cDNA Consortium; Poustka, A.; Wiemann, S. SMART amplification combined with cDNA size fractionation in order to obtain large full-length clones. BMC Genomics 2004, 5, 36.

14. Zhu, Y.Y.; Machleder, E.M.; Chenchik, A.; Li, R.; Siebert, P.D. Reverse transcriptase template switching: A SMART approach for full-length cDNA library construction. Biotechniques 2001, 30, 892-897.

15. Bogdanova, E.A.; Shagin, D.A.; Lukyanov, S.A. Normalization of full-length enriched cDNA. Mol. Biosyst. 2008, 4, 205-212. 
16. Sugahara, Y.; Carninci, P.; Itoh, M.; Shibata, K.; Konno, H.; Endo, T.; Muramatsu, M.; Hayashizaki, Y. Comparative evaluation of 5'-end-sequence quality of clones in CAP trapper and other full-length-cDNA libraries. Gene 2001, 263, 93-102.

17. Shagina, I.; Bogdanova, E.; Mamedov, I.Z.; Lebedev, Y.; Lukyanov, S.; Shagin, D. Normalization of genomic DNA using duplex-specific nuclease. Biotechniques 2010, 48, 455-459.

18. Zhulidov, P.A.; Bogdanova, E.A.; Shcheglov, A.S.; Vagner, L.L.; Khaspekov, G.L.; Kozhemyako, V.B.; Matz, M.V.; Meleshkevitch, E.; Moroz, L.L.; Lukyanov, S.A.; et al. Simple cDNA normalization using kamchatka crab duplex-specific nuclease. Nucleic Acids Res. 2004, 32, e37.

19. Pelaz, S.; Ditta, G.S.; Baumann, E.; Wisman, E.; Yanofsky, M.F. B and C floral organ identity functions require SEPALLATA MADS-box genes. Nature 2000, 405, 200-203.

20. Hake, S.; Smith, H.; Holtan, H.; Magnani, E.; Mele, G.; Ramirez, J. The role of KNOX genes in plant development. Annu. Rev. Cell Dev. Biol. 2004, 20, 125-151.

21. Bharathan, G.; Goliber, T.E.; Moore, C.; Kessler, S.; Pham, T.; Sinha, N.R. Homologies in leaf form inferred from KNOXI gene expression during development. Science 2002, 296, 1858-1860.

22. Hay, A.; Tsiantis, M. A KNOX family TALE. Curr. Opin. Plant Biol. 2009, 12, 593-598.

23. Adamczyk, B.J.; Lehti-Shiu, M.D.; Fernandez, D.E. The MADS domain factors AGL15 and AGL18 act redundantly as repressors of the floral transition in Arabidopsis. Plant J. 2007, 50, 1007-1019.

24. Amasino, R. Floral induction and monocarpic versus polycarpic life histories. Genome Biol. 2009, $10,228$.

25. Chatterjee, M.; Bermudez-Lozano, C.L.; Clancy, M.A.; Davis, T.M.; Folta, K.M. A strawberry KNOX gene regulates leaf, flower and meristem architecture. PLoS One 2011, 6, e24752-e24752.

26. Carninci, P.; Shibata, Y.; Hayatsu, N.; Sugahara, Y.; Shibata, K.; Itoh, M.; Konno, H.; Okazaki, Y.; Muramatsu, M.; Hayashizaki, Y. Normalization and subtraction of cap-trapper-selected cDNAs to prepare full-length cDNA libraries for rapid discovery of new genes. Genome Res. 2000, 10, 1617-1630.

27. Wells, C.A.; Ravasi, T.; Sultana, R.; Yagi, K.; Carninci, P.; Bono, H.; Faulkner, G.; Okazaki, Y.; Quackenbush, J.; Hume, D.A.; et al. Continued discovery of transcriptional units expressed in cells of the mouse mononuclear phagocyte lineage. Genome Res. 2003, 13, 1360-1365.

28. Wakimoto, B.T. Doubling the rewards: Testis ESTs for Drosophila gene discovery and spermatogenesis expression profile analysis. Genome Res. 2000, 10, 1841-1842.

29. Sakurai, T.; Plata, G.; Zapata, R.F.; Seki, M.; Salcedo, A.; Toyoda, A.; Ishiwata, A.; Tohme, J.; Sakaki, Y.; Shinozaki, K.; et al. Sequencing analysis of 20,000 full-length cDNA clones from cassava reveals lineage specific expansions in gene families related to stress response. BMC Plant Biol. 2007, 7, 66.

30. Lu, C.; Wallis, J.G.; Browse, J. An analysis of expressed sequence tags of developing castor endosperm using a full-length cDNA library. BMC Plant Biol. 2007, 7, 42.

31. Nagasaki, H.; Sakamoto, T.; Sato, Y.; Matsuoka, M. Functional analysis of the conserved domains of a rice KNOX homeodomain protein, OSH15. Plant Cell 2001, 13, 2085-2098. 
32. Cole, M.; Nolte, C.; Werr, W. Nuclear import of the transcription factor SHOOT MERISTEMLESS depends on heterodimerization with BLH proteins expressed in discrete sub-domains of the shoot apical meristem of Arabidopsis thaliana. Nucleic Acids Res. 2006, 34, 1281-1292.

33. Jouannic, S.; Collin, M.; Vidal, B.; Verdeil, J.L.; Tregear, J.W. A class I KNOX gene from the palm species Elaeis uineensis (Arecaceae) is associated with meristem function and a distinct mode of leaf dissection. New Phytol. 2007, 174, 551-568.

34. Cary, A.J.; Che, P.; Howell, S.H. Developmental events and shoot apical meristem gene expression patterns during shoot development in Arabidopsis thaliana. Plant J. 2002, 32, 867-877.

35. Zhang, S.; Wong, L.; Meng, L.; Lemaux, P.G. Similarity of expression patterns of knotted 1 and ZmLEC1 during somatic and zygotic embryogenesis in maize (Zea mays L.). Planta 2002, 215, 191-194.

36. Hay, A.; Barkoulas, M.; Tsiantis, M. ASYMMETRIC LEAVES1 and auxin activities converge to repress BREVIPEDICELLUS expression and promote leaf development in Arabidopsis. Development 2006, 133, 3955-3961.

37. Shani, E.; Burko, Y.; Ben-Yaakov, L.; Berger, Y.; Amsellem, Z.; Goldshmidt, A., Sharon, E.; Ori, N. Stage-specific regulation of Solanum lycopersicum leaf maturation by class 1 KNOTTED1-LIKE HOMEOBOX proteins. Plant Cell 2009, 21, 3078-3092.

38. Sakamoto, T.; Kamiya, N.; Ueguchi-Tanaka, M.; Iwahori, S.; Matsuoka, M. KNOX homeodomain protein directly suppresses the expression of a gibberellin biosynthetic gene in the tobacco shoot apical meristem. Genes Dev. 2001, 15, 581-590.

39. Jasinski, S.; Piazza, P.; Craft, J.; Hay, A.; Woolley, L.; Rieu, I.; Phillips, A.; Hedden, P.; Tsiantis, M. KNOX action in Arabidopsis is mediated by coordinate regulation of cytokinin and gibberellin activities. Curr. Biol. 2006, 15, 1560-1565.

40. Ng, M.; Yanofsky, M. Function and evolution of the plant MADS-box gene family. Nat. Rev. Genet. 2001, 2, 186-195.

41. Vandenbussche, M.; Theissen, G.; van de Peer, Y.; Gerats, T. Structural diversification and neo-functionalization during floral $M A D S$-box gene evolution by $C$-terminal frameshift mutations. Nucleic Acids Res 2003, 31, 4401-4409.

42. Chang, Y.Y.; Chiu, Y.F.; Wu, J.W.; Yang, C.H. Four orchid (Oncidium Gower Ramsey) AP1/AGL9-like MADS Box genes show novel expression patterns and cause different effects on floral transition and formation in Arabidopsis thaliana. Plant and Cell Physiol. 2009, 50, 1425-1438.

43. Delgado Sandoval, S.C.; Abraham Juárez, M.J.; Simpson, J. Agave tequilana MADS genes show novel expression patterns in meristems, developing bulbils and floral organs. Sex. Plant Reprod. 2012, 25, 11-26.

44. Tzeng, T.Y; Hsiao, C.C.; Chi, P.J.; Yang, C.H. Two lily SEPALLATA-Like genes cause different effects on floral formation and floral transition in Arabidopsis. Plant Physiol. 2003, 133, 1091-1101.

45. Honma, T.; Goto, K. Complexes of MADS-box proteins are sufficient to convert leaves into floral organs. Nature 2001, 409, 525-529. 
46. Theissen, G. Development of floral organ identity: Stories from the MADS house. Curr. Opin. Plant Biol. 2001, 4, 75-85.

47. Lee, J.H.; Yoo, S.J.; Park, S.H.; Hwang, I.; Lee, J.S.; Ahn, J.H. Role of SVP in the control of flowering time by ambient temperature in Arabidopsis. Genes Dev. 2007, 21, 397-402.

48. Michaels, S.D.; Bezerra, I.C.; Amasino, R.M. FRIGIDA-related genes are required for the winter-annual habit in Arabidopsis. Proc. Natl. Acad. Sci. USA 2004, 101, 3281-3285.

49. Hartmann, U.; Hohmann, S.; Nettesheim, K.; Wisman, E.; Saedler, H.; Huijser, P. Molecular cloning of SVP: A negative regulator of the floral transition in Arabidopsis. Plant J. 2000, 2, 351-360.

50. Kanno, A.; Nakada, M.; Akita, Y.; Hirai, M. Class B gene expression and the modified ABC model in nongrass monocots. The Sci. World J. 2007, 7, 268-279.

51. Goujon, M.; McWilliam, H.; Li, W.; Valentin, F.; Squizzato, S.; Paern J.; Lopez, R. A new bioinformatics analysis tools framework at EMBL-EBI. Nucleic Acids Res. 2010, 38, W695-W699.

(C) 2012 by the authors; licensee MDPI, Basel, Switzerland. This article is an open access article distributed under the terms and conditions of the Creative Commons Attribution license (http://creativecommons.org/licenses/by/3.0/). 